COLLEGE OF

AGRICULTURE \&

NATURAL RESOURCES

DEPARTMENT OF AGRICULTURAL AND

RESOURCE ECONOMICS

WP 17-07

\title{
Estimating Ex Ante Cost Functions for Stochastic Technologies
}

\author{
Robert G. Chambers \\ University of Maryland \\ Department of Agricultural and Resource Economics \\ Teresa Serra \\ Univsersity of Illinois \\ Department of Agricultural and Consumer Economics
}




\title{
Estimating Ex Ante Cost Functions for Stochastic Technologies
}

\author{
Robert G Chambers and Teresa Serra ${ }^{1}$
}

July 10, 2017

${ }^{1}$ Chambers: University of Maryland and School of Economics, University of Queensland. Serra: University of Illinois. 


\begin{abstract}
This paper revisits the problem of estimating ex ante cost functions previously studied by Pope and Just (1996) and Moschini (2001). An ex ante cost function that generalizes their ex ante cost functions is introduced, and an econometric procedure for estimating a flexible approximation to it is developed. That generalized cost function is economically relevant not only for the Just and Pope (1996) choice setting but for general producer risk preferences, general stochastic technologies, and general forms of price uncertainty. An econometric strategy for estimating the resulting cost structure that adapts Moschini's (2001) "fullinformation" approach is developed. And that is followed by an econometric application to US agriculture.
\end{abstract}

Key words: Econometric cost functions, stochastic decision environments, risk, uncertainty.

JEL codes: C51, C52, C31, D22, D24, Q11, Q12 
Ever since Nerlove's (1963) pathbreaking contribution, the econometric estimation of cost functions has been an integral part of applied production analysis. Econometrically estimated cost structures have significantly extended our empirical understanding of technological change, input substitutability, economies of size and scale, and economies of scope. Despite these successes, our ability to estimate cost structures for truly stochastic technologies remains relatively primitive. In that context, successes have been few, and applications frequently require restrictive assumptions on the technology and the underlying risk attitudes of producers.

A fundamental challenge to estimating cost structures for stochastic technologies is that cost-minimizing choices are often conditioned by variates that are unobservable to the econometrician. For example, Pope and Just (1996) and Moschini (2001) argue that a risk-neutral producer facing a stochastic technology and price uncertainty uncorrelated with production uncertainty minimizes the cost of producing expected output at the time production decisions are made and not the cost of producing observed output. Thus, econometric specifications of cost functions based on observed, rather than expected, output may suffer from specification error. Pope and Just (1996) and Moschini (2001) propose different approaches for tackling the resulting econometric problems.

This paper revisits the problem posed by Pope and Just (1996) and Moschini (2001). An ex ante cost function that generalizes their ex ante cost functions is introduced, and an econometric procedure for estimating a flexible approximation to it is developed. That generalized cost function is economically relevant not only for the Just and Pope (1996) choice setting but for general producer risk preferences, general stochastic technologies, and general forms of price uncertainty. Producers need not be risk-neutral. The stochastic technology need not be representable by a single-product stochastic production function. And output prices need not be nonstochastic or uncorrelated with production uncertainty.

To provide the economic rationale for this cost function, we first briefly consider the decision setting faced by producers. That setting differs from that in Pope and Just (1996) and Moschini (2001). Where Pope and Just (1996) and Moschini (2001) studied producers only having access to a stochastic technology to generate income, the current paper recognizes that producers have access to financial markets. That difference proves crucial to developing an econometric strategy for modelling cost structures. In that setting, rational production decisions under uncertainty must be consistent with minimizing the cost of producing stochastic revenue as discounted using a market-determined stochastic discount factor. Being market-determined, that stochastic discount factor affords a natural instrument on which to base estimation.

The plan of the paper is a follows. The next section details the decision setting and derives the ex ante cost function. It is shown to generalize those considered by Pope and Just (1996) and Moschini (2001). Once the economic relevance of the cost function is established, the paper then turns to econometric issues. An econometric strategy that adapts Moschini's (2001) "full-information" approach is developed. And that is followed by an econometric application to US agriculture using an updated version of the data set employed by Pope and Just (1996). The econometric results are discussed, and the paper then concludes. 


\section{The Decision Setting and the Ex Ante Cost Function}

There are two periods: period 0 (the decision period) involves no uncertainty and period 1 is uncertain. Uncertainty about period 1 is represented by the finite state space, $S$, and with a slight abuse of notation $S=(1,2, \ldots, S)$. The set of probability measures is denoted by $\Delta \subset \mathbb{R}_{+}^{S}$.

Following Chambers and Quiggin (2000) and Moschini (2001), the stochastic production technology is represented by a continuous input correspondence that maps stochastic output, $z \in \mathbb{R}_{+}^{S}$, into sets of inputs, chosen nonstochastically in period 0, capable of producing it. For notational convenience we treat the case where the producer operates a single-product technology. The results extend directly to the multiple-output case. Denote the inputs by $x \in \mathbb{R}_{+}^{N}$ and their period 0 prices, which are nonstochastic, by $w \in \mathbb{R}_{+}^{N}$. The continuous input correspondence describing the technology, $X: \mathbb{R}_{+}^{S} \rightrightarrows \mathbb{R}_{+}^{N}$, is defined

$$
X(z) \equiv\left\{x \in \mathbb{R}_{+}^{N}: x \text { can produce } z \in \mathbb{R}^{S}\right\}
$$

$X(z)$ satisfies: $X(z)+\mathbb{R}_{+}^{N} \subset X(z)$ (free disposability of inputs); $z^{\prime} \geq z \Longrightarrow X\left(z^{\prime}\right) \subset X(z)$ (free disposability of output); and $X(z)+\lambda\left(X\left(z^{\prime}\right)-X(z)\right) \subset X\left(z+\lambda\left(z^{\prime}-z\right)\right)$ for $\lambda \in(0,1)$ (convexity of the graph).

For $q \in \mathbb{R}^{S}$ define the ex ante input correspondence, $X^{q}: \mathbb{R}_{+} \rightrightarrows \mathbb{R}_{+}^{N}$, by

$$
X^{q}(r) \equiv\left\{x \in X(z): r \leq q^{\top} z\right\}
$$

where $q^{\top}=\left(q_{1}, \ldots, q_{S}\right) .{ }^{1}$ Interpreting $q$ as (period 0$)$ state-claim prices for period 1 stochastic income, $X^{q}(r)$ gives the input combinations capable of producing a state-claim revenue $r \in \mathbb{R}$. $X^{q}$ is continuous and satisfies: $X^{q}(r)+\mathbb{R}_{+}^{N} \subset X^{q}(r)$ (free disposability of inputs); $r^{\prime} \geq r \Longrightarrow X^{q}\left(r^{\prime}\right) \subset X^{q}(r)$ (free disposability of $r$ ); $X^{q}(r)=X^{q}(r)+\lambda\left(X^{q}(r)-X^{q}(r)\right)$ for $\lambda \in(0,1)$ (convexity of the graph) and $X^{\mu q}(\mu r)=X^{q}(r)$ for $\mu>0$ (homogeneity of degree zero). The first three properties are inherited directly from the properties of $X$ and the fourth, homogeneity of degree zero, ensures that the units in which one chooses to denominate period 1 state-contingent returns does not affect the underlying production possibilities.

Financial markets are stochastic and beyond the control of the individual producer. The matrix of $J$ ex ante financial security returns in period 1 is $R \in \mathbb{R}^{S \times J}$, which is of full column rank. Each asset's period 0 price is normalized at 1 . The producer's period 0 portfolio vector is denoted $m \in \mathbb{R}^{J}$, and the linear sub space spanned by $R$ is denoted $M \subset \mathbb{R}^{S}$. Define

$$
P \equiv\left(R^{\top} R\right)^{-1} R^{\top}
$$

We assume that no arbitrages exist.

Producer preferences over period 0 consumption, $y_{0} \in \mathbb{R}_{+}$, and period 1 stochastic consumption, $y \in \mathbb{R}_{+}^{S}$, are given by the continuous and strictly monotonic $W\left(y_{0}, y\right)$. Given a period 0 predetermined wealth of $i_{0} \in \mathbb{R}_{+}$, the producer's period 0 problem is, therefore,

$$
\max _{z, m, y_{0}, y, x}\left\{W\left(y_{0}, y\right): y_{0} \leq i_{0}-w^{\top} x-1^{\top} m, y \leq p \cdot z+R m, x \in X(z)\right\},
$$

\footnotetext{
${ }^{1}$ For $a \in \mathbb{R}^{S}$ and $b \in \mathbb{R}^{S}, a^{\top} b$ represents the inner product in terms of the Euclidean norm.
} 
where $1^{\top}$ is a $J$ dimensional row vector of ones, and the notation $x \cdot y \in \mathbb{R}^{S}$ denotes the element-wise product of $x \in \mathbb{R}^{S}$ and $y \in \mathbb{R}^{S}$.

Because $W$ is strictly monotonic, in any interior producer equilibrium the period 0 and period 1 budget constraints must bind and, therefore,

$$
\begin{aligned}
y_{0} & =i_{0}-w^{\top} x-1^{\top} m \\
y & =p \cdot z+R m .
\end{aligned}
$$

Thus,

$$
R m=y-p \cdot z
$$

requiring that $y-p \cdot z \in M$. The unique portfolio solving the period 1 budget constraint given $y$ and $z$ is

$$
m=P(y-p \cdot z)
$$

Substituting this result into the period 0 budget constraint and that result into (1) reduces the producer's problem to:

$$
\max _{z, y, x}\left\{W\left(i_{0}-w^{\top} x-1^{\top} P(y-p \cdot z), y\right): x \in X(z)\right\} .
$$

The random variable $1^{\top} P \in \mathbb{R}^{S}$ is the ideal stochastic discount factor for all $y \in M$. It prices, in period 0 units any period 1 asset falling in $M$ (for example, Cochrane 2001). Denote

$$
q^{* \top} \equiv\left(1^{\top} P\right) \cdot p
$$

Thus, the sth element of $q^{* \top} \in \mathbb{R}^{S}$ corresponds to the sth element of $1^{\top} P$ times the sth element of $p$, and $q^{* \top} z=1^{\top} P(p \cdot z)$ corresponds to the stochastically discounted revenue from producing $z \in \mathbb{R}_{+}^{S}$.

By Bellman's optimality principle and the definition of $X^{q}$, the producer problem now decomposes in steps as

$$
\begin{gathered}
\max _{y}\left\{W\left(i_{0}+\max _{z, x}\left\{q^{* \top} z-w^{\top} x: x \in X(z)\right\}-1^{\top} P y, y\right)\right\}, \\
\max _{y}\left\{W\left(i_{0}+\max _{r, x}\left\{r-w^{\top} x: x \in X^{q^{*}}(r)\right\}-1^{\top} P y, y\right)\right\}
\end{gathered}
$$

and

$$
\max _{y}\left\{W\left(i_{0}+\max _{r}\left\{r-\min _{x}\left\{w^{\top} x: x \in X^{q^{*}}(r)\right\}\right\}-1^{\top} P y, y\right)\right\}
$$

The subproblem

$$
c^{*}\left(w, q^{*}, 1\right) \equiv \max _{r}\left\{r-\min _{x}\left\{w^{\top} x: x \in X^{q^{*}}(r)\right\}\right\}
$$

requires that $x$ be chosen to minimize the period 0 cost of producing discounted revenue $r$ for the stochastic discount factor $q^{*}$ and that $r$ be chosen to maximize discounted profit. For that reason, $c^{*}\left(w, q^{*}, 1\right)$ is referred to as the discounted profit function in what follows.

This decomposition reveals that producer behavior can be modeled as though the producer pursues a three-stage optimization procedure. First, the cost of producing revenue discounted using $q^{*}$ is minimized. 
Then discounted profit from production is maximized by chosing discounted revenue, $r$, properly. This ensures that period 0 income, which is risk-free, is as large as possible for any level of period-1 consumption-risk exposure. Put more simply, all riskless opportunities (arbitrages) to raise consumption are fully exploited. In the final stage, the producer solves the intertemporal consumption allocation problem out of an available period 0 income of $i_{0}+c^{*}\left(w, q^{*}, 1\right)$.

Following Pope and Just (1996) and Moschini (2001), define the first-stage ex ante cost function, $c(w, q, r)$, as the period 0 minimal cost of producing $q$-discounted revenue, $r$. That is,

$$
c(w, q, r) \equiv \min \left\{w^{\top} x: x \in X^{q}(r)\right\},
$$

if $X^{q}(r) \neq \emptyset$ and $\infty$ otherwise. $c(w, q, r)$ is: superlinear and nondecreasing in $w$, convex and nondecreasing in $r$, homogeneous of degree zero in $(q, r)$, and nonincreasing and quasi-concave in $q .{ }^{2}$ Moreover, under our assumptions, the following duality exists between it and the ex ante input correspondence:

$$
X^{q}(r)=\cap_{w \in \mathbb{R}_{+}^{N}}\left\{x: c(w, q, r) \leq w^{\top} x\right\} .
$$

If one defines the convex conjugate of $c(w, q, r)$ in $u \in \mathbb{R}$ by

$$
c^{*}(w, q, u)=\sup _{r}\{u r-c(w, q, r)\}
$$

it's easily seen that the solution to the discounted profit maximization problem corresponds to $c^{*}(w, q, u)$ evaluated at $q=q^{*}$ and $u=1$. Moreover, under our assumptions, standard conjugate duality (Rockafellar $1970)$ implies $c(w, q, r)$ can be recovered by applying the conjugate mapping to $c^{*}(w, q, u)$, that is,

$$
\begin{aligned}
c(w, q, r) & =\sup _{u}\left\{u r-c^{*}(w, q, u)\right\} \\
& =c^{* *}(w, q, r) .
\end{aligned}
$$

The ex ante cost function studied by Pope and Just (1996) and Moschini (2001) is the special case of $c(w, q, r)$ where $q=\delta \bar{p} \pi$ with $\delta \in \mathbb{R}_{++}$is an intertemporal discount factor, $\bar{p} \in \mathbb{R}_{++}$is a nonstochastic output price (or the mean of a stochastic output price whose distribution is independent of that for $z$ ), and $\pi \in \Delta$ is a probability measure. ${ }^{3}$ The exact relationship is clarified by exploiting the zero homogeneity of $c(w, q, r)$ to obtain

$$
c(w, \delta \bar{p} \pi, r)=c\left(w, \pi, \frac{r}{\delta \bar{p}}\right)
$$

\footnotetext{
${ }^{2}$ To establish quasi-concavity, let$$
\hat{x} \in \arg \min \left\{w^{\top} x: x \in X^{q^{o}+\mu\left(q^{o}-q^{\prime}\right)}(r)\right\},
$$

for $\mu \in(0,1)$. To ensure that it can produce a $z$ such that $\left[q^{o}+\mu\left(q^{o}-q^{\prime}\right)\right]^{\top} z \geq r, \hat{x}$ has to belong to either $X^{q^{o}}$ or $X^{q^{\prime}}$. And thus,

$$
c\left(w, q^{o}+\mu\left(q^{o}-q^{\prime}\right), r\right) \geq \min \left\{c\left(w, q^{o}, r\right), c\left(w, q^{\prime}, r\right)\right\}
$$

${ }^{3}$ Both Pope and Just (1996) and Moschini (2001) operate in a single-period context so that their $\delta$ is implicitly set to one. Moreover, neither study includes a specific notation for the probability measure underlying expectation formation in their $e x$ ante cost structures.
} 
which gives the cheapest way to produce an expected output equalling $\frac{r}{\delta \bar{p}}$. Its conjugate dual is

$$
c^{*}(w, \delta \bar{p} \pi, 1)=\sup _{r}\{r-c(w, \delta \bar{p} \pi, r)\}
$$

which is the expected profit function for $\pi \in \Delta$. It is well-known that $c^{*}(w, \delta \bar{p} \pi, 1)$ possesses the same properties in $(w, \delta \bar{p} \pi)$ as a profit function developed for a nonstochastic technology. Moschini (2001) uses this observation to suggest that an optimal expected value retrieved from $c^{*}(w, \delta \bar{p} \pi, 1)$ is an appropriate platform upon which to base estimation of an econometric representation of the stochastic technology.

Besides $c(w, \delta \bar{p} \pi, r)^{\prime} s$ specific reliance on risk neutrality and on restrictions on the stochastic nature of $p$ (see Pope and Just 1996) other distinctions exist between it and $c\left(w, q^{*}, r\right)$. Most importantly, $c\left(w, q^{*}, r\right)$ depends on $q^{*} \in \mathbb{R}^{S}$ which is determined by market forces beyond the producer's control. The former, however, depends upon $\delta$ and $\pi$. These depend upon the producer's preferences and beliefs about the relative likelihood of different states of nature occurring. Moschini (2001) clearly recognizes this aspect of his ex ante cost structure when he writes it "...reflects the producers' expectations in addition to the technological properties of the stochastic production function". And, thus, it remains perfectly plausible that individual producers with different expectation mechanisms would possess different ex ante cost structures. This is to be contrasted with the fact that $c\left(w, q^{*}, r\right)$ is economically relevant for any producer with strictly monotonic preferences.

\section{On Econometric Estimation}

The good news is that a cost function that is relevant for a general decision environment exists. The bad news is that the discounted revenue, $r$, that conditions $c\left(w, q^{*}, r\right)$ is not observable. The econometric problem is analogous to that faced by Pope and Just (1996) and Moschini (2001). In their case, the econometrician only has observations on realizations of stochastic output, $z(s)$, for a single $s \in S$ and not on expected output. In the present case, observations are available for realized revenue, $p(s) z(s)$ for realized $s$, but not for $r=q^{* \top} z$.

To fix ideas, let $c_{\theta}(w, q, r)$ be a parametrized version of $c(w, q, r)$, with $\theta$ the vector of parameters to be estimated. Given smoothness in $w$, Shephard's Lemma and standard econometric practice (see, for example, expression (9) in Moschini (2001)) suggests estimation be based on the input-demand system:

$$
X=\nabla_{w} c_{\theta}\left(w, q^{*}, r\right)+\varepsilon
$$

where $X \in \mathbb{R}_{+}^{N}$ is the vector of observed input demands, $\nabla_{w} c_{\theta}\left(w, q^{*}, r\right)$ is the gradient of $c_{\theta}\left(w, q^{*}, r\right)$ in $w$, and $\varepsilon$ is the error vector for the input demands.

Because $r$ is unobservable and jointly dependent, an econometrically tractable replacement must be found. Simply replacing $r$ with $p(s) z(s)$ for realized $s$ is clearly inappropriate, a point emphasized by both Pope and Just (1996) and Moschini (2001). Pope and Just (2001) and Moschini (2001) suggest different 
approaches for resolving the resulting econometric problem. ${ }^{4}$ We follow Moschini's (2001) "full-information" approach and replace $r$ with

$$
r_{\theta}\left(w, q^{*}, 1\right) \in \arg \max _{r}\left\{r-c_{\theta}\left(w, q^{*}, r\right)\right\}
$$

to obtain the form:

$$
X=\nabla_{w} c_{\theta}\left(w, q^{*}, r_{\theta}\left(w, q^{*}, 1\right)\right)+\varepsilon .
$$

The practical argument for the "full-information" solution is that $c_{\theta}\left(w, q^{*}, r\right)$ is of economic interest because producers solve the discounted profit problem. And because $r_{\theta}\left(w, q^{*}, 1\right)$ solves that problem, $r_{\theta}\left(w, q^{*}, 1\right)$ "...provides the correct nonlinear mapping for..." the instruments $\left(w, q^{*}\right)$ (Moschini 2001). A potential sticking point is that this approach requires either closed-form solutions for $r_{\theta}\left(w, q^{*}, 1\right)$ or that numerical solutions for it be "....retrieved as part of the estimation routine" (Moschini 2001).

If Nerlove's (1963) approach is followed, and one starts with $X^{q}(r)$ and proceeds via (3), finding closedform solutions for $\nabla_{w} c_{\theta}\left(w, q^{*}, r\right)$ and $r_{\theta}\left(w, q^{*}, 1\right)$ may prove impractical. ${ }^{5}$ But that is only one option. Following in the spirit of Gorman (1968), econometric production-system specifications have routinely been based on dual rather than primal representations of the technology. After all, the mathematical essence of the dual approach is that problems posed in one context can be exhaustively characterized by analyzing their natural duals. Thus, unless the analyst is being awarded points for "degree of difficulty", the most efficient approach is to formulate problems in terms of variables that all agents face and on which we have, or can construct, observations. So, the current problem can be framed in terms of the prices $\left(w, q^{*}\right)$, cost, discounted profit, and revenue.

Straightforward dual alternatives to $X^{q}(r)$ are readily available. One is to work with a parametric specification of $c(w, q, r)$ that admits a closed-form solution to the problem:

$$
c_{\theta}^{*}\left(w, q^{*}, 1\right)=\sup _{r}\left\{r-c_{\theta}\left(w, q^{*}, r\right)\right\} .
$$

Another is to work with the conjugate dual of $c_{\theta}\left(w, q^{*}, r\right), c_{\theta}^{*}\left(w, q^{*}, 1\right)$, and use (5) and Hotelling's lemma to obtain an econometrically tractable representations of $r_{\theta}\left(w, q^{*}, 1\right)$ and $x_{n}\left(w, q^{*}, r_{\theta}\left(w, q^{*}, 1\right)\right)$.

An example illustrates the first. The parametric cost structure

$$
c_{\theta}^{Q}(w, q, r)=\alpha_{\theta}(w, q)+\frac{r}{A_{\theta}(w, q)}+\frac{1}{2}\left(\frac{r}{B_{\theta}(w, q)}\right)^{2},
$$

\footnotetext{
${ }^{4}$ Pope and Just (1996) suggest retrieving their version of $r_{\theta}\left(w, q^{*}, 1\right)$ (expected output) as part of the estimation process. In the current notation, their approach retrieves $r$ as the solution to the following problem

$$
\max \left\{r: \inf _{w}\left\{1-c(w, q, r)+w^{\top} x\right\} \geq 1\right\}
$$

as part of the estimation process. This is equivalent to solving for the distance function associated with $X^{q}(r)$ as evaluated at $x$ and then choosing the maximal $r$ associated with that distance function. Moschini (2001) criticizes this approach on a number of grounds including the potential presence of simultaneous-equations bias and an ultimate lack of consistency.

${ }^{5}$ Both Pope and Just (1996) and Moschini (2001) start with a stochastic production function and not $X^{q}(r)$. However, Pope and Just (1996) use their stochastic production structure to induce an equivalent distance function representation. That alternative, if one chooses, is also available here.
} 
can be parametrized to approximate an arbitrary smooth $c(w, q, r)$ flexibly. The closed-form solution for $r_{\theta}\left(w, q^{*}, 1\right)$ is

$$
r_{\theta}^{Q}\left(w, q^{*}, 1\right)=B_{\theta}\left(w, q^{*}\right)\left(1-A_{\theta}\left(w, q^{*}\right)^{-1}\right),
$$

which when combined with Shephard's Lemma yields derived demands of the form:

$$
\begin{aligned}
x_{n}\left(w, q^{*}, r_{\theta}\left(w, q^{*}, 1\right)\right) & =\frac{\partial \alpha_{\theta}\left(w, q^{*}\right)}{\partial w_{n}}-\frac{B_{\theta}\left(w, q^{*}\right)\left(1-A_{\theta}\left(w, q^{*}\right)^{-1}\right)}{A_{\theta}\left(w, q^{*}\right)^{2}} \frac{\partial A_{\theta}\left(w, q^{*}\right)}{\partial w_{n}} \\
& -\frac{\left(B_{\theta}\left(w, q^{*}\right)\left(1-A_{\theta}\left(w, q^{*}\right)^{-1}\right)\right)^{2}}{B_{\theta}\left(w, q^{*}\right)^{3}} \frac{\partial B_{\theta}\left(w, q^{*}\right)}{\partial w_{n}}
\end{aligned}
$$

for $n=1, . ., N$.

Expression (7) provides a platform upon which to base econometric estimation of the parameters of a flexible representation of the ex ante cost structure. Typically, however, it will result in a parametric specification where the parameters of the cost structure, $\theta$, enter the estimating equations nonlinearly. Our empirical example pursues the alternative tack and starts with an econometrically tractable representation of

$$
x\left(w, q^{*}, r_{\theta}\left(w, q^{*}, 1\right)\right) \equiv \nabla_{w} c_{\theta}\left(w, q^{*}, r_{\theta}\left(w, q^{*}, 1\right)\right) .
$$

Hotelling's Lemma and (5) are then used to induce $c_{\theta}(w, q, r)$. The specific form chosen is the linear-inparameters form

$$
x_{n}\left(w, q^{*}, r_{\theta}\left(w, q^{*}, 1\right)\right)=\sum_{j=1}^{N} \beta_{n j}\left(\frac{w_{j}}{w_{n}}\right)^{\frac{1}{2}}+\frac{1}{k} \sum \gamma_{s n}\left(\frac{q_{s}^{*}}{w_{n}}\right)^{k}, \quad n=1, \ldots, N,
$$

with $\beta_{k j}=\beta_{j k}$. This system of derived demands corresponds to the discounted-profit maximizing demands associated with the cost structure

$$
c_{\theta}(w, q, r)=2 \sum_{n=1}^{N} \sum_{j=1}^{N} \beta_{n j}\left(w_{j} w_{n}\right)^{\frac{1}{2}}+\frac{k-1}{k}\left(\frac{1}{1-k} \sum_{s} \sum_{k} \gamma_{k s} q_{s}^{k} w_{k}^{1-k}\right)^{\frac{1}{1-k}} r^{\frac{k}{k-1}},
$$

which permits the existence of non-parametric returns to scale and elasticities of substitution.

That (9) generates (8) as the discounted-profit maximizing demands is verified in stages. Applying Shephard's lemma to (9) gives the cost minimizing demands as

$$
x_{n}\left(w, q^{*}, r\right)=\sum_{j=1}^{N} \beta_{n j}\left(\frac{w_{j}}{w_{n}}\right)^{\frac{1}{2}}+\frac{1}{k} r^{\frac{k}{k-1}}\left(\frac{1}{1-k} \sum_{s} \sum_{k} \gamma_{k s} q_{s}^{* k} w_{k}^{1-k}\right)^{\frac{k}{1-k}} \sum_{s} \gamma_{n s}\left(\frac{q_{s}^{*}}{w_{n}}\right)^{k} .
$$

Solving the discounted profit maximization problems gives

$$
r_{\theta}\left(w, q^{*}, 1\right)=\frac{1}{1-k} \sum_{s} \sum_{k} \gamma_{k s} q_{s}^{* k} w_{k}^{1-k}
$$

as the solution for optimal discounted revenue. Substituting this result into (10) gives (8). 


\section{An Empirical Application}

Following Pope and Just (1996, 1998), our empirical analysis is based on agricultural data drawn from the United States Department of Agriculture (USDA) data set "Indices of farm output, input, and total factor productivity for the United States, 1948-2013", currently available on line at: http://www.ers.usda.gov/ data-products/agricultural-productivity-in-the-us.aspx. These data consist of observations on aggregate agricultural input use, their associated price indexes, aggregate agricultural output and its price index for the United States for 1948-2013. The input aggregates considered were capital (K), labor (L), and intermediate materials (I). Pope and Just (1996, 1998) used earlier versions (1948-1989) of this data set. Other studies using different versions of these data include Ball $(1985,1988)$, Vasavada and Chambers (1986), Chavas (2008), and Yang and Shumway (2016). We also used data on financial returns obtained from the dataset "Historical Returns: Stocks, T. Bonds \& T. Bills" available from http://www.stern.nyu . edu/ ${ }^{\sim}$ adamodar/New_Home_Page/data.html.

There are several practical issues to be tackled in constructing an econometric version of our model using these data. First, econometric estimation of the input demand system requires an empirical approximation for each time period for $q^{*} \in \mathbb{R}^{S}$, which is composed of $\left(1^{\top} P\right)$ and the stochastic output price $p$. These are constructed by assuming that market returns at each time period can be reasonably represented by the rate of return of the S\&P500 index $\left(r_{t}\right)$ and the 10-year T-Bond $\left(a_{t}\right)$. Details on the exact procedure used in constructing this approximation are provided in the next section.

The second practical problem arises from the time-series nature of our agricultural data set. Previous studies using the aggregate data on agricultural netputs and their prices, apart from including parameters to capture the effect of technical progress, have typically paid little attention to the time-series characteristics of these data. If we followed that approach, we might simply estimate an input-demand structure of the form

$$
X_{n t}=\sum_{j=1}^{N} \beta_{n j}\left(\frac{w_{j t}}{w_{n t}}\right)^{\frac{1}{2}}+\frac{1}{k} \sum_{s} \gamma_{s n}\left(\frac{q_{s t}^{*}}{w_{n t}}\right)^{k}+\mu_{n} t+\varepsilon_{n t}, \quad n=1, \ldots, N,
$$

where $X_{n t}$ denotes the observed value of the $n t h$ demand at time $t, w_{n t}$ denotes the observed value of the $n t h$ input price at time $t, q_{s t}^{*}$ denotes the observed value of $q_{s}^{*}$ at time $t, \mu_{n}$ measures the effect of technical progress on the optimal usage of the $n t h$ demand, and $\varepsilon_{n t}$ is an econometric error term.

Such a procedure will yield consistent estimates of the parameters if the underlying data series are suitably stationary. As a practical matter, that does not appear to be the case. Dickey and Fuller (1979) and Elliott et al. (1996) unit root tests suggest the presence of unit roots in all the dependent input quantity variables, $X_{n t}$, and most of the transformed variables (for example, square roots of the normalized input prices and the powers of the normalized output prices). ${ }^{6}$ As usual in the time-series econometrics literature, practicality thus requires that our input-demand system be estimated in first difference form. ${ }^{7}$ Thus, our

\footnotetext{
${ }^{6}$ Results are not presented here but are available from the authors upon request.

${ }^{7}$ Unit root tests performed on first differenced data show no remaining stochastic trends.
} 
practical solution is to use the following econometric representation:

$$
X_{n t}-X_{n t-1}=\mu_{n}+\sum_{j \neq n} \beta_{n j}\left[\left(\frac{w_{j t}}{w_{n t}}\right)^{\frac{1}{2}}-\left(\frac{w_{j t-1}}{w_{n t-1}}\right)^{\frac{1}{2}}\right]+\frac{1}{k} \sum \gamma_{s n}\left[\left(\frac{q_{s t}^{*}}{w_{n t}}\right)^{k}-\left(\frac{q_{s t-1}^{*}}{w_{n t}-1}\right)^{k}\right]+\nu_{n t}
$$

where $\nu_{n t}$ is the econometric error structure. This estimation strategy precludes direct estimation of the location parameter $\left(\beta_{n n}\right)$ for each derived demand. Hence, we cannot fully recapture the parameters associated with our cost structure. It is important to emphasize, however, that this problem is not a consequence of modelling the underlying production system as stochastic rather than deterministic. Rather, it arises from the time-series nature of the data set.

\section{Generating the Random Variates Corresponding to $S$}

Time-series econometric procedures are used to identify a probability model for each financial returns series and the agricultural output price $\left(R_{t}, A_{t}\right.$ and $p_{t}$, respectively). Assuming that $S_{t}$ (that is, the set of states of nature for year $t$ ) is time invariant and can be generated from the probability distribution characterizing variability over time, empirical approximations to $\left(R_{t}, A_{t}, p_{t}\right)^{\top} \in \mathbb{R}^{3 S}$ for each sample year $t$ can then be simulated by combining those results with Monte Carlo methods.

The conditional mean models that best represent the data are developed using Box-Jenkins (Box et al., 1994) methods. The first step is to test for the presence of unit roots in each individual time series. This is investigated by applying Augmented Dickey Fuller (ADF) (Dickey and Fuller, 1979) and Elliott et al. (1996) unit-root tests. While the S\&P500 and 10-year T-Bond series are found to be stationary, the output-price series has a stochastic trend and requires first-differencing of the series.

After preliminary testing, a stationary conditional mean model was fitted to each of the three series separately by identifying an optimal autoregressive-moving average (ARMA) model. Results, presented in Table 1 below, show that no ARMA effects characterize the S\&P500 returns. It consists of white noise fluctuating around a positive constant. The 10-year T-Bond is found to be characterized by a structural break around 1970, which is captured through the introduction of a dummy variable in the conditional mean model. No evidence of ARMA effects is found in either series. Finally, the first differenced log output price has a MA(1) pattern. Prices are expressed in logs to induce normality. Goodness of fit checks are conducted in order to ensure adequate modelling. The Lagrange Multiplier (LM) tests for serial correlation and autoregressive conditional heteroscedasticity (ARCH) effects (Asteriou and Hall, 2011) suggest no serial correlation and no ARCH effects in any of the three models' residual series. Moreover, the Jarque-Bera (1981) normality test suggests that the innovation distributions are normal.

The estimated time-series models were then used with Monte Carlo simulations to construct approximations to the underlying random variables. For each series and for each time-period in the sample $(t=$ 1948 to 2013), 500 random draws are produced from the normal distribution. Responses to the shocks are obtained from each conditional mean model. Because the conditional mean models for financial returns do not have ARMA effects, responses need to be recursively generated only for the output price model. In this 
case, for each of the 500 simulations associated with period $t$, the presample response is taken to be fixed and to correspond to the observation at time $t$ ( $t=1948$ to 2013), the time for which the state space is being generated. The output price response is then transformed in order to convert changes of the logged price index to price index levels.

Figures 1-3 depict the average, as well as the 5th and 95th percentiles, generated for the S\&P500 returns, the 10-year T-bond returns, and the output price index (in levels) for each sample period. Figure 4 presents the mean and the $5^{t h}$ and $95^{t h}$ percentiles derived from computing $q_{t}^{*}$ for each year.

\section{Input demand system}

In principle, the state space, $S$, can be quite large, and given our procedure for approximating the random variates, one ostensibly could set the cardinality of $S$ to 500. But, as a practical matter, there exists a clear trade off between the fineness of the partition for $S$ and parametric parsimony. For practical econometric reasons, we have thus elected to partition into two coarse events, thought of as high and low. The random variate for the low (high) event is computed as the average of the values of $q_{t}^{*}$ below (above) the median. Results are denoted as $q_{L}$ and $q_{H}$, respectively and presented in Figure 5.

In our empirical model, we set $k=2$ and input prices are obtained from the USDA dataset in the form of price indices. The capital input price index is represented by $w_{K}$. It includes durable equipment, service buildings, land and inventories. The intermediate inputs price index is representeded by $w_{I}$ and corresponds to the costs of feed and seed, energy, fertilizer and lime, pesticides, purchased services and other intermediate inputs. The third input price is the labor price index $\left(w_{L}\right)$ that includes the cost of hired workers and self-employed and unpaid family. The corresponding input quantity indices for each of these input price categories are denoted by $X_{K}, X_{I}$ and $X_{L}$.

We estimate two versions of the model. One treats the agricultural technology as stochastic and follows the estimation strategy developed above. The second treats agricultural output as nonstochastic and replaces the random variate $\left(q_{L}^{*}, q_{H}^{*}\right)$ with the (singleton) observed aggregate output price. When the models were fit with a non-autoregressive error structure, the Hosking (1981) variant of the multivariate Q statistic indicated the presence of serial correlation in the residuals of the model requiring the addition of an autoregressive term in each of the three equations (that is, one lag of the dependent variable). ${ }^{8}$

The resulting parameter estimates are presented in Table 2. (Parameter estimates obtained while ignoring the time-series characteristics of the data are reported in the Appendix.) In viewing both the stochastic and non-stochastic versions of the model, several results are apparent. First, both specifications are characterized by a relatively large number of imprecisely estimated parameters. ${ }^{9}$ Second, the stochastic version of the model

\footnotetext{
${ }^{8}$ Annual data rarely have ARCH properties. This was confirmed through LM tests for ARCH effects.

${ }^{9}$ This contrasts starkly with previous analyses using versions of these data that have ignored their time-series characteristics. However, results reported in an Appendix verify that both non-stochastic and stochastic versions of the model fit while ignoring the time-series characteristics of the data report very (apparently) precise parameter estimates. Our remaining analysis, however, is based upon the results reported in Table 2.
} 
suggests that producers respond to output price variability differentially according to the state of Nature in which it arises. Thus, the stochastic specification suggests that capital demand is non-regressive in $q_{H}^{*}$ but regressive in $q_{L}^{*}$, that intermediate input demand is regressive in $q_{H}^{*}$ but non-regressive $q_{L}^{*}$, and that labor demand is non-regressive in $q_{H}^{*}$ and $q_{L}^{*}$. The non-stochastic specification, on the other hand suggests that all input demands are nonregressive in the output price.

The pattern of differential responses to $q_{H}^{*}$ and $q_{L}^{*}$ observed in the capital and intermediate-input demand systems can be partially explained by considering a simple thought experiment. Suppose that the length of the interval $\left[q_{L}^{*}, q_{H}^{*}\right]$ were to increase as a result of increasing $q_{H}^{*}$ and decreasing $q_{L}^{*}$ by an equal amount. The results in Table 2 would suggest that this increase in price dispersion, which may be identified with either greater price risk or price uncertainty, would lead to more capital-intensive production practices. Not only that, but the magnitude of the estimated parameters would suggest that the increase associated with falling prices in the low-state would be larger than the increase prompted by the increase in the high state. Thus, producers respond to increased price uncertainty by expanding capital usage, and they respond more forcefully in this regard to a downward extension of the lower tail of the distribution than to an upward extension of the upper tail.

The results also suggest that intermediate input usage plays just the reverse role. Intermediate input usage increases as the interval $\left[q_{L}^{*}, q_{H}^{*}\right]$ decreases and decreases as the interval increases. And again we see that the response is relatively greater to changes in the low-state price than to changes in the high-state output prices. Labor usage, on the other hand, responds positively to increases in both the high and low-state discounted output price, but because it appears to respond more forcefully to changes in the high-state price than to changes in the low-state, lengthening the interval $\left[q_{L}^{*}, q_{H}^{*}\right]$ in the manner suggested would lead to more intensive labor usage. Conversely, shrinking the interval and moving to a more concentrated price dispersion would lead to decreased labor usage. Thus, capital and labor both appear to be risk complementary, in regards to price risk, in the sense of Chambers and Quiggin (2000), while intermediate materials appear to be risk subsitutes.

Both the stochastic and the non-stochastic versions of the model suggest that technical change in aggregate US agriculture has been capital and labor saving and intermediate input using. These results contrast with studies of aggregate US agriculture based on earlier time periods (see, for example, Just and Pope 1996 and the sources cited therein) that suggest that technical change in agriculture is generally capital using and labor saving. Two points are relevant here. First, it is true that US agriculture went through a period of intensive capital investment between the end of the Second World War and approximately 1980. Since approximately 1983 (the last 30 years of our sample), however, there has been a secular decline in aggregate capital usage in US agriculture. Second, it must be remembered that previous empirical examinations of US agriculture have not taken account of the time-series characteristics of the underlying data. And thus, results on the nature of the character of technical change are not directly comparable to ours.

Summary results on calculated elasticities of input demands with respect to input prices are presented in 
Table 3. The elasticities reported there are the immediate or impact elasticities. Elasticities were computed for each sample period, using observed demands. The means and standard deviations as well as the coefficient of variation for each of these elasticities are the results reported in Table 3. Several points are worth remarking. First, when compared to previous studies of aggregate US agriculture, with the exception of the average own-price elasticity for intermediate inputs for the non-stochastic specification, the average elasticities are extremely low and indicative of little or no price responsiveness. Second, again with the exception of the own-price elasticity for intermediate inputs, the rough magnitude of the elasticities for both the stochastic and the non-stochastic specifications are broadly similar. And third, the results suggest that in the main the aggregate inputs are substitutes for one another. The sole exception is capital and intermediates in the nonstochastic specification.

\section{Concluding Remarks}

We have reconsidered the problem posed by Pope and Just (1996) and Moschini (2001) of estimating ex ante cost functions for stochastic technologies. In doing so, we have generalized the decision setting considered by those authors to allow producers to have access to capital and equity markets and to face arbitrary forms of price uncertainty. Both generalizations seem eminently plausible for producers operating in a modern economy with active financial markets. An ex ante cost function that generalizes their ex ante cost functions is introduced, and an econometric procedure for estimating a flexible approximation to it is developed. That generalized cost function is economically relevant not only for the Just and Pope (1996) choice setting, which requires risk-neutral producers facing a production technology characterized by a stochastic production function, but also for general producer risk preferences, general stochastic technologies, and general forms of

price uncertainty. An econometric version of the resulting ex ante cost function has been estimated for US agriculture. 


\section{References}

Asteriou, D., and S. G. Hall. Applied Econometrics (Second Edition). 2011: Palgrave Macmillan, New York.

Ball, V. E. "Modeling Supply Response in a Multiproduct Framework." American Journal of Agricultural Economics 70 (1988): 813-25.

Ball, V. E. "Output, Input, and Productivity Measurement in U.S. Agriculture, 1948-79." American Journal of Agricultural Economics 67 (1985): 475-586.

Bera, A. K., and C. M. Jarque. "An Efficient Large Simple Test for Normality of Observations and Regression Residuals." Australian National University Working Papers in Econometrics 40, 1981.

Box, G. E. P., G. M Jenkins, and G. C. G. C. Reinsel. Time Series Analysis: Forecasting and Control (Third Edition). Englewood Cliffs, NJ: Prentice Hall, 1994.

Chambers, R. G., and J. Quiggin. Uncertainty, Production, Choice, and Agency: The State-Contingent Approach. New York: Cambridge University Press, 2000.

Chavas, J.-P. "A Cost Approach to Economic Analysis Under State-Contingent Production Uncertainty." American Journal of Agricultural Economics 90 (2008): 435-46.

Cochrane, J. H. Asset Pricing. Princeton: Princeton University Press, 2001.

Dickey, D. A., and W. A. Fuller. "Distribution of the Estimators for Autoregressive Time Series Regressions With a Unit Root." Journal of the American Statistical Association 74 (1979): 427-31.

Elliott, G., T. J. Rothenberg, and J. H. Stock. "Efficient Tests for an Autoregressive Unit Root." Econometrica 64 (1996): 813-36.

Gorman, W. M. "Measuring the Quantities of Fixed Factors." Value, Capital and Growth. ed. J. N. WolfeEdinburgh: Edinburgh University Press, 1968.

Hosking, J. M. R. " Equivalent Forms of the Multivariate Portmanteau Statistic." . Journal of the Royal Statistical Society - Series B 43, no. 2 (1981): 261-62.

Moschini. G. "Production Risk and the Estimation of Ex Ante Cost Functions." Journal of Econometrics 100 (2001): 357-80.

Nerlove, M. L. "Returns to Scale in Electricity Supply." Measurement in Economics: Studies in Mathematical Economics and Econometrics in Memory Yehuda Grunfeld. C. L. Christ et al.Stanford : Stanford University Press, 1963.

Pope, R. D., and R. E. Just. "Cost Function Estimation Under Risk Aversion." American Journal of Agricultural Economics 80 (1998): 296-302.

—. "Empirical Implementation of Ex Ante Cost Functions." Journal of Econometrics 72 (1996): 231-49.

Rockafellar, R. T. Convex Analysis. Princeton: Princeton University Press, 1970.

Vasavada, Utpal, and Robert G. Chambers. "Investment in U.S. Agriculture." American Journal of Agricultural Economics 68, no. 4 (1986): 950-960.

Yang, S., and C. R. Shumway. "Dynamic Adjustment in US Agriculture Under Climate Change." American Journal of Agricultural Economics 98 (2016): 910-924. 
Table 1. Box-Jenkins method

\begin{tabular}{|c|c|c|c|}
\hline & S\&P500 & $\begin{array}{l}\text { 10-year T- } \\
\text { Bond }\end{array}$ & $\begin{array}{l}\text { Logged output } \\
\text { price changes }\end{array}$ \\
\hline \multicolumn{4}{|l|}{ Conditional mean model parameters } \\
\hline Constant & $\begin{array}{l}0.127 \\
(0.021)^{* *}\end{array}$ & $\begin{array}{l}0.018 \\
(0.018)\end{array}$ & $\begin{array}{l}0.019 \\
(0.014)\end{array}$ \\
\hline Dummy $(=1$ if $t>1970)$ & & $\begin{array}{l}0.060 \\
(0.022)^{* *}\end{array}$ & \\
\hline MA(1) & & & $\begin{array}{l}0.585 \\
(0.102)^{* *}\end{array}$ \\
\hline JB test for normality & 2.035 & 2.914 & 4.828 \\
\hline LM test for correlation up to order 5 & 1.205 & 1.058 & 1.409 \\
\hline LM test for ARCH up to order 5 & 0.859 & 1.301 & 0.813 \\
\hline
\end{tabular}


Table 2. Input demand system when the time-series properties of the data are considered

\begin{tabular}{|c|c|c|c|c|c|c|c|}
\hline \multicolumn{4}{|c|}{ STOCHASTIC MODEL } & \multicolumn{4}{|c|}{ NON-STOCHASTIC MODEL } \\
\hline & $x_{\mathbf{K}}$ & $x_{\mathrm{I}}$ & $x_{\mathrm{L}}$ & & $x_{K}$ & $x_{I}$ & $x_{\mathrm{L}}$ \\
\hline \multirow[t]{2}{*}{$q_{H}$} & 8.60 & -116.38 & $26.88^{* *}$ & $p$ & $1291.95 * *$ & $33315.52 * *$ & $1144.40 * *$ \\
\hline & $(9.72)$ & $(149.63)$ & $(7.05)$ & & $(558.22)$ & $(7,034.31)$ & (362.44) \\
\hline \multirow[t]{2}{*}{$q_{L}$} & -36.49 & 514.32 & 5.84 & & & & \\
\hline & $(37.54)$ & $(474.30)$ & (19.69) & & & & \\
\hline$w_{\mathrm{K}}$ & & & & $w_{K}$ & & & \\
\hline \multirow[t]{2}{*}{$w_{I}$} & 1094.45 & & & $w_{\text {I }}$ & $-3,233.02$ & & \\
\hline & $(1689.405)$ & & & & $(2,293.69)$ & & \\
\hline \multirow[t]{2}{*}{$w_{\mathbf{L}}$} & $1,997.12$ & $12828.64 * *$ & & $w_{\mathbf{L}}$ & $3284.2694 * *$ & $8,029.94$ & \\
\hline & $(1,522.54)$ & $(5,536.04)$ & & & $(1,618.98)$ & $(6,225.26)$ & \\
\hline \multirow[t]{2}{*}{ intercept } & -99.01 & $1141.84^{*}$ & $-1160.89^{* *}$ & intercept & -91.26 & $1475.40 * *$ & $-1191.98 * *$ \\
\hline & $(88.58)$ & $(586.97)$ & $(448.95)$ & & $(88.81)$ & $(512.60)$ & $(482.58)$ \\
\hline \multirow[t]{2}{*}{$\mathbf{A R}(\mathbf{1})$} & $0.53^{* *}$ & -0.02 & 0.12 & $\operatorname{AR}(\mathbf{1})$ & $0.55^{* *}$ & 0.07 & $0.18^{*}$ \\
\hline & $(0.09)$ & $(0.12)$ & $(0.10)$ & & $(0.09)$ & $(0.11)$ & $(0.10)$ \\
\hline $\begin{array}{l}\text { MVQSTAT up to } \\
\text { order } 5\end{array}$ & 47.24 & & & $\begin{array}{l}\text { MVQSTAT up to } \\
\text { order } 5\end{array}$ & 46.40 & & \\
\hline
\end{tabular}

order 5

Note: $q_{L}$ and $q_{H}$ take very small values leading to very big coefficient estimates. To prevent this, they were rescaled (by taking the sum of values above and below the median instead). Elasticity estimates do not change. Standard errors in parethesis 
Table 3. Input demand elasticities

\begin{tabular}{|c|c|c|c|c|c|c|}
\hline & \multicolumn{2}{|c|}{ STOCHASTIC } & \multicolumn{4}{|c|}{ NON-STOCHASTIC } \\
\hline & mean & std & cv & mean & std & cv \\
\hline EK_wK & -0.026 & 0.006 & -0.209 & -0.034 & 0.019 & -0.577 \\
\hline EK_wI & 0.008 & 0.001 & 1.307 & -0.025 & 0.003 & -0.130 \\
\hline EK_wL & 0.011 & 0.004 & 0.350 & 0.018 & 0.006 & 0.350 \\
\hline EI_WK & 0.005 & 0.001 & 0.187 & -0.014 & 0.003 & -0.187 \\
\hline EI_wI & 0.000 & 0.021 & 120.820 & -0.535 & 0.272 & -0.509 \\
\hline EI_WL & 0.040 & 0.004 & 0.096 & 0.025 & 0.002 & 0.096 \\
\hline EL_WK & 0.015 & 0.004 & 0.246 & 0.024 & 0.006 & 0.246 \\
\hline EL_wI & 0.098 & 0.016 & 0.160 & 0.061 & 0.010 & 0.160 \\
\hline $\mathbf{E} \mathbf{L}_{-} \mathbf{w}_{\mathbf{L}}$ & -0.226 & 0.105 & -0.464 & -0.207 & 0.092 & -0.442 \\
\hline
\end{tabular}

Note: the models labeled cross-sectional are the models that ignore the time-series properties of the data. 
Figure 1. Summary statistics for the state-space generated for S\&P 500 returns for each year

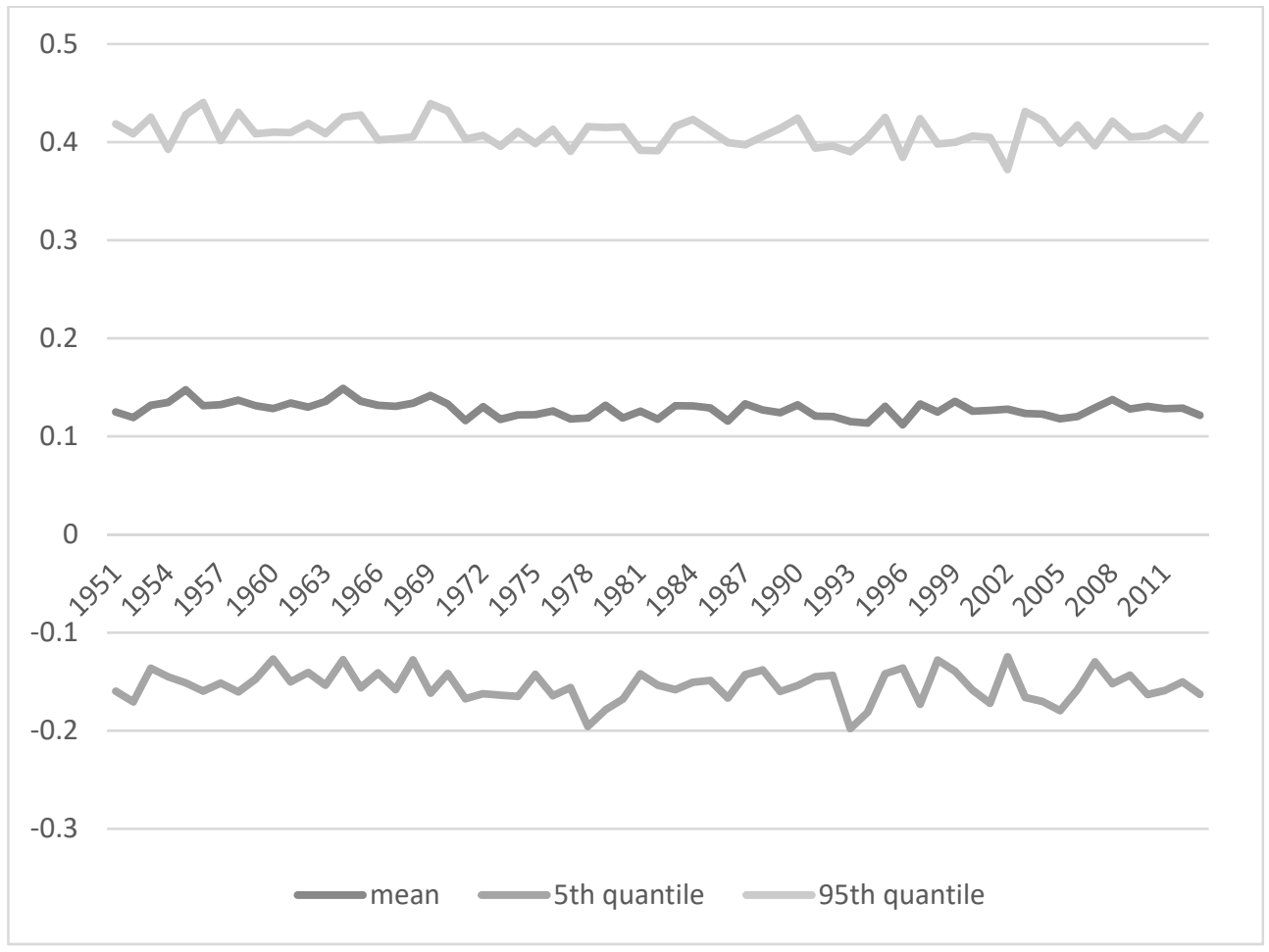


Figure 2. Summary statistics for the state space generated for the 10-year T-Bond returns for each year

\section{0 year T-Bond}

0.3

0.25

0.2

0.15

0.1

0.05

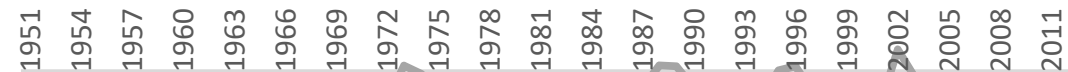
$-0.05$

$-0.1$

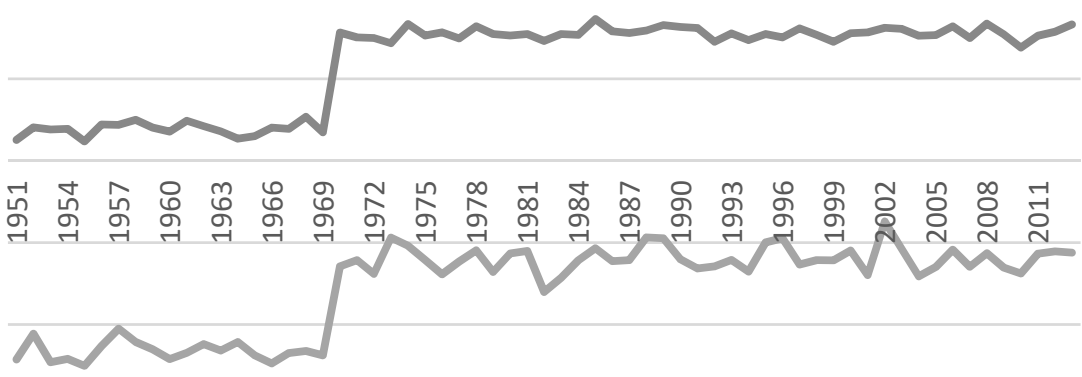

$-0.15$

$\longrightarrow$ mean $\longrightarrow$ th quantile $\longrightarrow$ th quantile 
Figure 3. Summary statistics for the state space generated for the output price index for each year

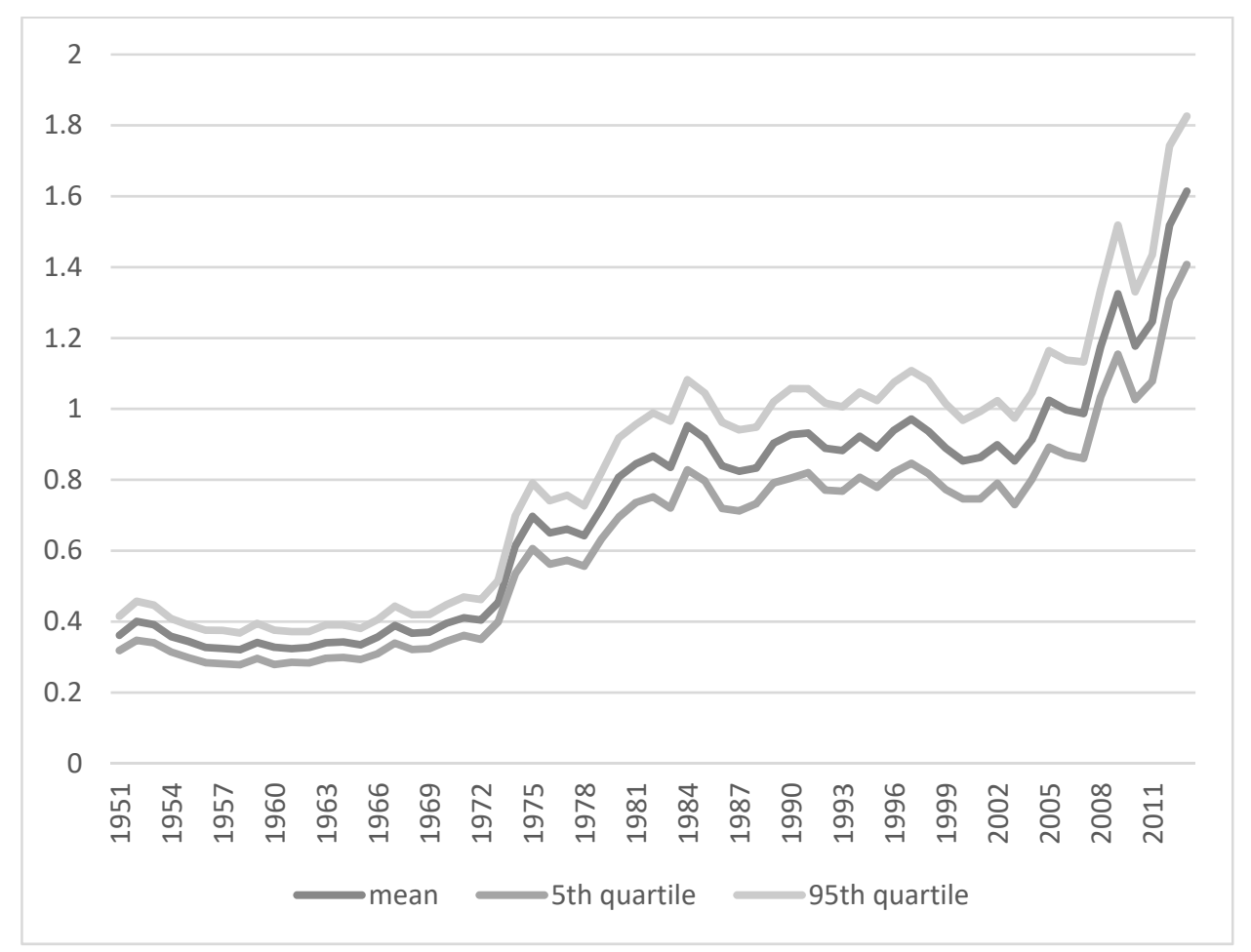


Figure 4. Summary statistics for the state space generated for $q_{t}^{* \mathrm{~T}}$ for each year

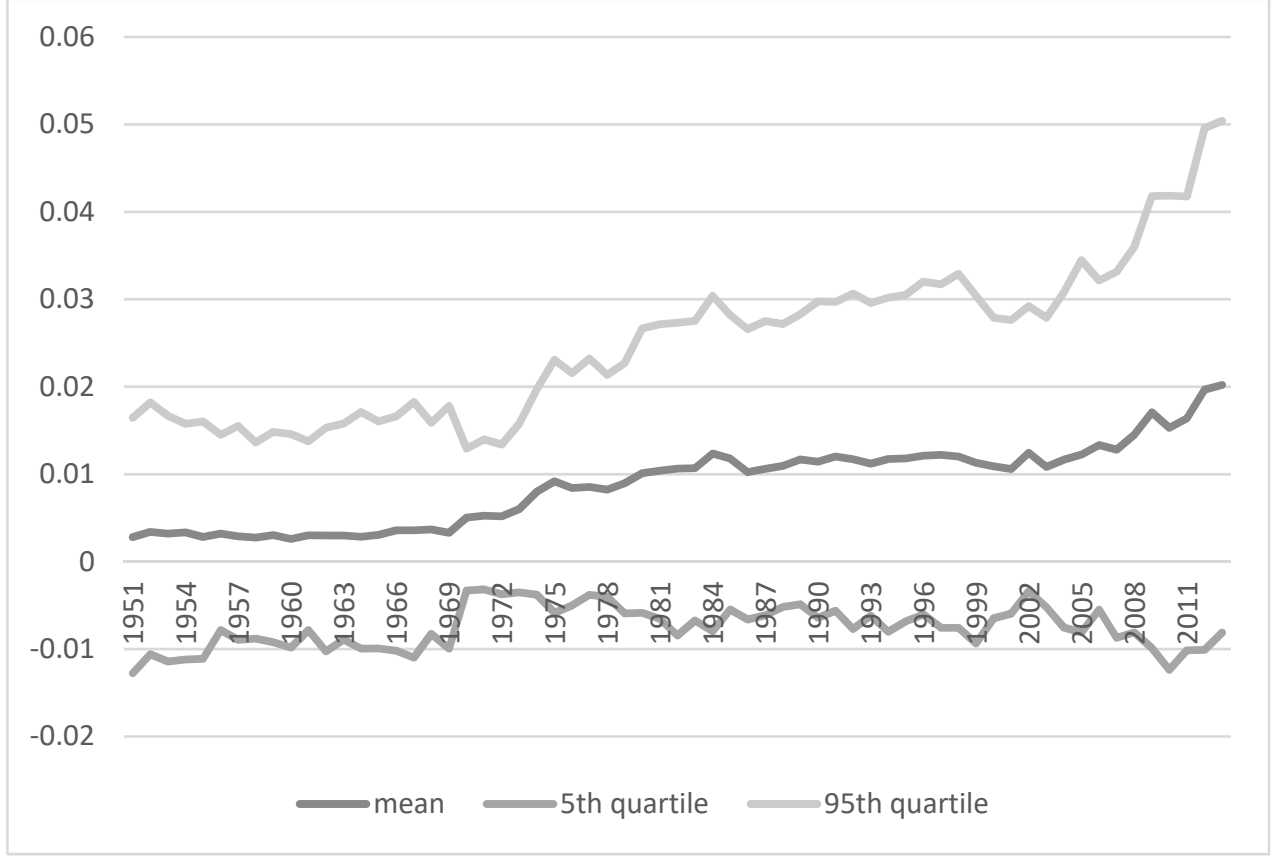


Figure 5. Coarse partition of the state space $q_{t}{ }^{* \mathrm{~T}}$ for each year

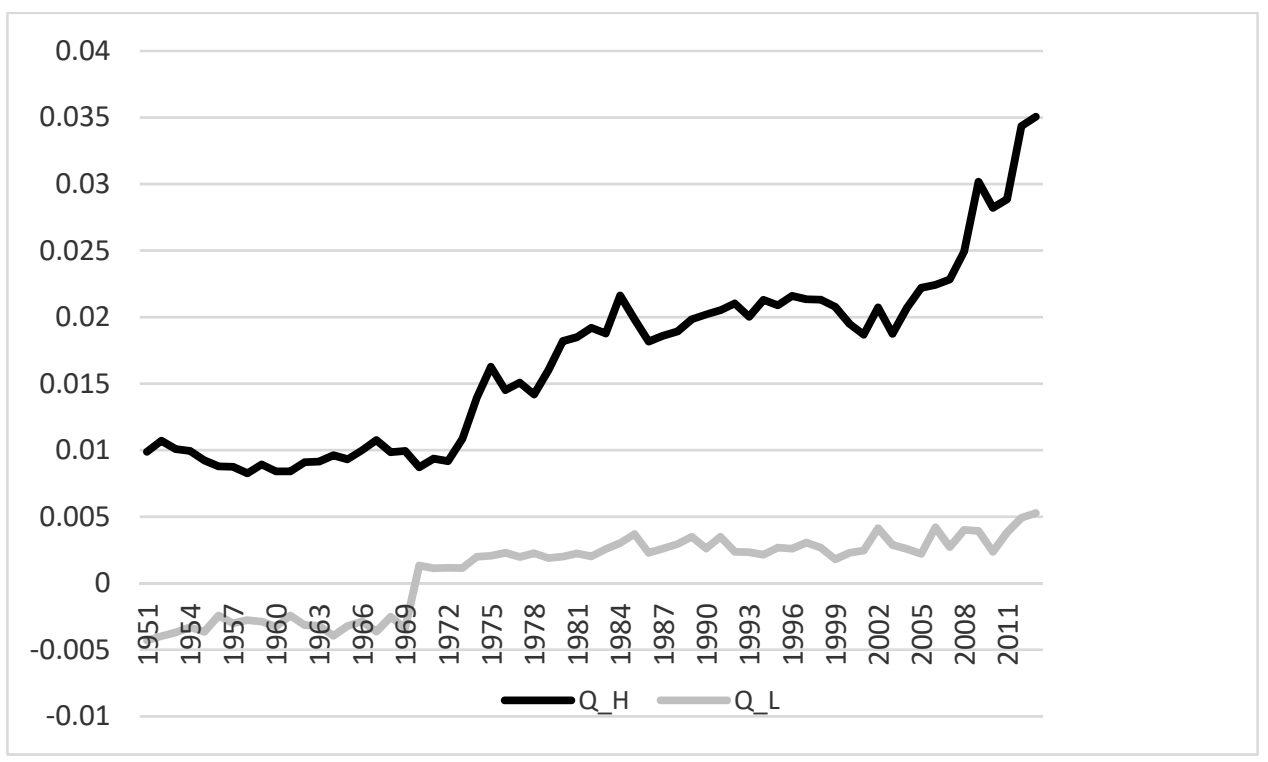




\section{APPENDIX}

Input demand system when time-series properties of the data are ignored

\begin{tabular}{|c|c|c|c|c|c|c|c|}
\hline \multicolumn{4}{|c|}{ STOCHASTIC MODEL } & \multicolumn{4}{|c|}{ NON-STOCHASTIC MODEL } \\
\hline & $x_{K}$ & $x_{\mathrm{I}}$ & $x_{\mathrm{L}}$ & & $x_{\mathbf{K}}$ & $x_{I}$ & $x_{\mathbf{L}}$ \\
\hline \multirow[t]{2}{*}{$q_{H}$} & $79.83^{*}$ & -140.21 & $31.66^{* *}$ & $p$ & $3950.58 * *$ & $4,506.06$ & $2353.88 * *$ \\
\hline & $(44.40)$ & $(198.12)$ & $(6.41)$ & & $(1,748.37$ & $(7,848.95)$ & (121.81) \\
\hline \multirow[t]{2}{*}{$\boldsymbol{q}_{L}$} & $-885.57 * *$ & -887.81 & $110.42 * *$ & & & & \\
\hline & $(220.13)$ & $(698.36)$ & $(35.92)$ & & & & \\
\hline \multirow[t]{2}{*}{$w_{\mathrm{K}}$} & $83041.64 * *$ & & & $w_{\mathrm{K}}$ & $78550.40^{* *}$ & & \\
\hline & $(2,807.81)$ & & & & $(3,006.89$ & & \\
\hline \multirow[t]{2}{*}{$w_{\mathbf{I}}$} & 5438.61 & $71680.86^{* *}$ & & $w_{\text {I }}$ & $2,460.60$ & $57025.46^{* *}$ & \\
\hline & (3900.88) & $(7,849.87)$ & & & $(5,108.58$ & $(9,265.63)$ & \\
\hline \multirow[t]{2}{*}{$w_{\mathbf{L}}$} & $-14626.24 * *$ & $22088.48 * *$ & $118587.22^{* *}$ & $w_{\mathbf{L}}$ & $-21219.39 * *$ & $17381.62 * *$ & $133805.89^{* *}$ \\
\hline & $(3,162.15)$ & $(4,720.25)$ & $(9,806.35)$ & & $(3,750.94$ & $(4,422.88)$ & $(10,282.53)$ \\
\hline \multirow[t]{2}{*}{ trend } & $-274.98 * *$ & $785.26^{* *}$ & $-1127.80^{* *}$ & trend & -37.06 & $1107.64^{* *}$ & $-1207.87 * *$ \\
\hline & $(46.21)$ & $(125.46)$ & $(80.47)$ & & $(54.85$ & (135.58) & (80.78) \\
\hline
\end{tabular}

Note: $q_{L}$ and $q_{H}$ take very small values leading to very big coefficient estimates. To prevent this, they were rescaled (by taking the sum of values above and below the median instead). Elasticity estimates do not change. Standard errors are in parenthesis. 
Input demand elasticities when time-series properties of the data are ignored

\begin{tabular}{|c|c|c|c|c|c|c|}
\hline & \multicolumn{3}{|c|}{ STOCHASTIC } & \multicolumn{3}{|c|}{ NON-STOCHASTIC } \\
\hline & mean & std & cv & mean & std & cv \\
\hline EK_wK & 0.030 & 0.056 & 1.865 & -0.025 & 0.094 & -3.703 \\
\hline EK_wI & 0.042 & 0.005 & 0.130 & 0.019 & 0.002 & 0.130 \\
\hline EK_wL & -0.081 & 0.028 & -0.350 & -0.117 & 0.041 & -0.350 \\
\hline EI_WK & 0.024 & 0.004 & 0.187 & 0.011 & 0.002 & 0.187 \\
\hline EI_wI & 0.034 & 0.118 & 3.503 & -0.135 & 0.038 & -0.280 \\
\hline EI_WL & 0.068 & 0.007 & 0.096 & 0.054 & 0.005 & 0.096 \\
\hline EL_WK & -0.108 & 0.027 & -0.246 & -0.157 & 0.034 & -0.218 \\
\hline EL_wI & 0.168 & 0.027 & 0.160 & 0.132 & 0.021 & 0.160 \\
\hline EL_W $\mathbf{W}_{\mathbf{L}}$ & -0.233 & 0.203 & -0.873 & -0.225 & 0.221 & -0.980 \\
\hline
\end{tabular}

Note: the models labeled cross-sectional are the models that ignore the time-series properties of the data. 